\title{
Corrupted packets discarding mechanism to alleviate congestion in wireless body area network
}

\author{
Wan Aida Nadia Wan Abdullah ${ }^{1}$, Naimah Yaakob ${ }^{2}$, R. Badlishah Ahmad ${ }^{3}$, \\ Mohamed Elshaikh Elobaid ${ }^{4}$, Siti Asilah Yah ${ }^{5}$ \\ ${ }^{1,2,4,5}$ ENAC, School of Computer and Communication Engineering (SCCE), \\ Universiti Malaysia Perlis (UniMAP), Malaysia \\ ${ }^{3}$ Faculty of Informatics and Computing, Universiti Sultan Zainal Abidin (UniSZA), Malaysia
}

\begin{tabular}{l}
\hline \hline Article Info \\
\hline Article history: \\
Received Sep 25, 2018 \\
Revised Nov 26, 2018 \\
Accepted Jan 25, 2019 \\
\hline
\end{tabular}

\section{Keywords:}

Bit error rate

Congestion

Corrupted

ECG

WBAN

\begin{abstract}
Generation of high traffic from continuous sensing and collection of medical data from various biosensors on multiple body is most likely to occur in the Wireless Body Area Network (WBAN). This could be a factor to the congestion in the network. Occurrence of congestion would collapse the performances in the WBAN network in terms of increment in delay, high packets loss, reduction in throughput and packet deliver ratio (PDR). The crucial concerns in WBAN are prevention from the loss of critical data and longer delay in the network as they could result to late delivery of medical treatment and possibility of the increase in mortality. Therefore, this study proposes a mechanism to alleviate the congestion from happening in the first place through discarding the corrupted packets before the beginning of data transmission to the base station. Extensive simulations are done in $\mathrm{OMNeT}+$ to analyze the performance of the proposed mechanism by varying traffic from low to high under different number of nodes and constant Bit Error Rate (BER) and packet size. From the finding, it can be concluded that the proposed mechanism shows better performances in terms of low delay and packet loss as well as high throughput and PDR compared to typical WBAN.
\end{abstract}

Copyright $@ 2019$ Institute of Advanced Engineering and Science. All rights reserved.

\section{Corresponding Author:}

Wan Aida Nadia Wan Abdullah, ENAC, School of Computer and Communication Engineering (SCCE), Universiti Malaysia Perlis (UniMAP), 02600 Arau, Perlis, Malaysia.

Email: aidanadia@studentmail.unimap.edu.my

\section{INTRODUCTION}

A progressive revolution of intelligent bio-sensors and Internet technology have been a great help to the development of the Wireless Body Area Network (WBAN) or also known as Wireless Body Sensor Network (WBSN) technology nowadays. WBAN is one of the advanced technologies derived from Wireless Sensor Network (WSN) which is specially focused to the healthcare applications. This technology is formed through collection of various vital signals of the body such as Electrocardiogram (ECG), blood pressure, and temperature that are monitored and detected from specific bio-sensors. These bio-sensors can be wear, attached or implanted in the body. Later, these collected data will be sent to the hospital database through Internet in order to inform the medical authorities about the condition of the patients [1], [2]. Therefore, WBAN needs a timely delivery transmission and avoidance of any packet loss during transmission of these collected vital signals in order to reduce risk of mortality among patients. Also, this could enhance the reliability of the WBAN. However, transferring a bulk of these collected data simultaneously could result in network congestion that would prevent the fulfilment of the aforementioned requirements. 
Network congestion occurs due to several factors such as uncontrolled load of traffics, many-to-one communication (sensors to sink) and constrained resources such as bandwidth and buffer occupancy [3]-[5]. For example, sudden high traffic or data generation would occur in the network during the physiological signals sensing from the body. This is especially true when there is detection of abnormality in the reading of the vital signals from multiple patients. The affected bio-sensors should quickly transmit the abnormal data to the base station. This has severely resulted to the occurrence of packets collision during contending for medium access. In addition, some corrupted packets may exist in the transmission of bulk medical data which could as well bring to the network congestion. Not only that it causes congestion, but also might waste the scarce resources of the network such as bandwidth and energy. Specifically, the corrupted packets will be finally discarded at the receiver (base station). These discarded packets need to be retransmitted especially if it involved critical medical data. However, retransmission will increase the delay and consume more energy. To be brief, network congestion leads to high number of losses and dropped packets, high delay as well as low throughput and Packet Delivery Ratio (PDR). Hence, it is a must to design a new mechanism to control the network congestion in the first place.

This paper is aimed to analyze the impact of earlier discarding of the corrupted medical packets in terms of delay, percentage packet loss, throughput and PDR in order to avoid congestion in the WBAN network. The aforementioned network metrics are measured through two types of traffics from low to high with different number of nodes under constant Bit Error Rate (BER) and packet size. Section 2 reviews the existing congestion avoidance techniques in WBAN. Section 3 delivers method in conducting the experiments. Section 4 elaborates the significant findings from the proposed mechanism on the network performance of the WBAN. Section 5 concludes and delivers future works of this study.

\section{RELATED WORKS}

A number of studies have been carried out to design proper mechanisms for controlling congestion in WBAN through transport layer as it supports congestion control, flow control and fair bandwidth allocation [1], [6]. Congestion control can be categorized into three types which are congestion detection, congestion notification and congestion avoidance [5]. In particular, this section highlights on the existing mechanisms of congestion avoidance in WBAN. Congestion avoidance can be done via rate control, dropping packets, redirection of traffics, polite gossip policy and optimization of cross-layer design [7], [8].

The congestion avoidance schemes in [9], [10] have integrated the concept of Learning Automata (LA) through placing an intelligent automaton in every node. This automaton comprises a predefined set of action to continuously interact with surrounding. According to [9], the occurrence of the congestion can be prevented earlier since intermediate node itself contains complete information of the traffic at any instances and regulation for better incoming flow rate can take place. Optimal data rate is chosen by learning from past performance of the flow in the network through the computation of dropped packet. In other words, the rate's flow with least number of dropped packets will be chosen as optimal rate. This protocol equalized the packet arrival rate and packet service rate in all existing intermediate nodes to avoid large queuing at the nodes which is indirectly reduce congestion.

However, prevention of congestion is done through deployment of Active Queue Management (AQM) by creating separate virtual queues on a single queue in [10]. The input packet from child's node is stored in the queue based on source's traffic priority. Acceptance or drop of packet is decided by calculating packet drop probability. Once the packet is accepted, virtual queues and automata based Congestion Detection (ACD) will be implemented to control congestion. Automata work in three states which are normal, congestion avoidance and congested. Congestion level (CL) is the output of ACD. If CL exceeds the predefined threshold, Optimized Rate Adjustment (ORA) will take place. Factors of bandwidth's availability and level of priority are considered in optimizing the sending rates among the child's node to the parent's node.

The issue of available bandwidth is also being considered in congestion avoidance protocol addressed by [11] through integration of Relaxation Theory (RT) with Max-Min Fairness (MMF) mechanism at each intermediate node. This scheme earns better Quality of Services (QoS) specifically during high traffic generation from multiple nodes. RT is used to regulate packet's transmission rate. The awaiting packet will be served according to the Control Transmission (EL) which refers to the number of slots based on available bandwidth. Balancing sending rate among congested link is done by MMF through sharing same resources allocation. In specific, reduction in sending rate among source nodes that have higher sending rate so that sending rate of other source nodes with lower sending rate can be increased. When the value of EL is higher than available bandwidth, the EL for all sources will be reset to zero and new sending rate is applied. This mechanism still serves excessive incoming packets and ensures those packets reach to the destination although their transmission will be postponed or relaxed within tolerable delay compared to other 
mechanisms which consider those excessive packets to be lost at the end of data transmission. From the aforementioned methods, it can be seen that most of them considered about rate control to utilize the bandwidth and queue in controlling the congestion.

Conversely, this study concerns on earlier discarding of the corrupted packets in order to mitigate the congestion in the network. This mechanism is carried out before the transmission of the packets to the base station is initiated. This is because transmitting the corrupted packets would waste the scarce network resources because at the end of the process, these packets still would be discarded. Further elaboration on the proposed mechanism is done in following section.

\section{METHODOLOGY}

As aforementioned in Section I, this study emphasizes on the effects of discarding the corrupted medical packets of ECG before they are being transmitted to the base station. Corrupted packets can be defined as packets that have one or more bits in errors. Bit error rate (BER) refers to the ratio of number of bits received in error to the total number of bits received in the specific time as in [12] and shown in 1 as follows.

$$
\text { Bit Error Rate }(B E R)=\frac{\text { Number of bits in error }}{\text { Total number of bits received }}
$$

According to [13], [14], ECG sensors should have BER within the range of $10^{-10}$ to $10^{-3}$. To justify, this study chooses and sets value of high BER which is $10^{-3}$. To the fact, ECG sensors generate more packets especially during monitoring multiple bodies in a longer period of time. The Packet Error Rate (PER) also can be derived from BER since transmission of incorrectly bits could degrade the entire packets. This correlated relationship is indicated in 2 as in [15].

$$
\text { Packet Error Rate }(P E R)=1-(1-B E R)^{n}
$$

where $\mathrm{n}$ is the number of packet length in bits.

Based on 2, the output would be PER of 0.2 under fixed packet size of 30 bytes in this study. The overall flowchart and algorithm of the proposed mechanism in WBAN is depicted in Figure 1 and Table 1 as follows.

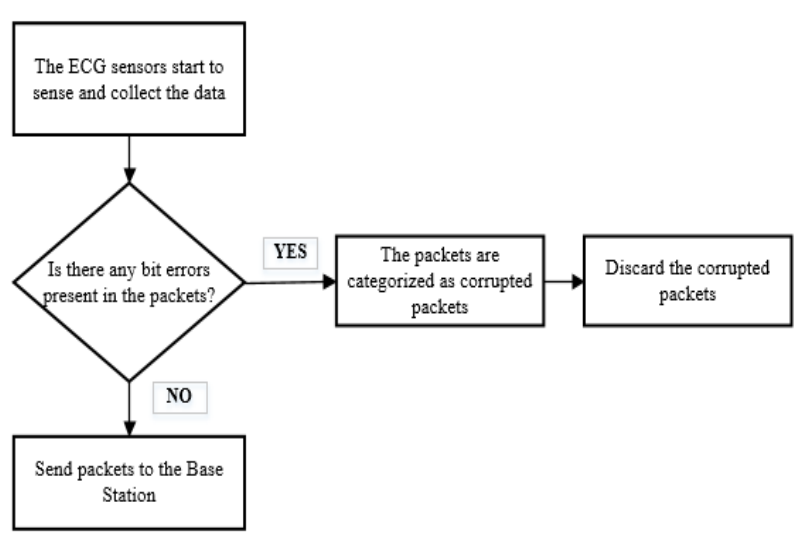

Table 1. Algorithm of the Proposed Mechanism

\begin{tabular}{lc}
\hline \multicolumn{2}{l}{ Algorithm 1: Overall proposed mechanism } \\
\hline 1. & Data: Packet size of ECG data (30 bytes) \\
2. & If (Packet Size $<=30$ bytes), then \\
3. & Packet $=$ ACCEPT ; \\
4. & \\
5. & If ( BER of Packets $=10^{-3}$, then, \\
6. & Packet $=$ CORRUPTED \&\& DISCARD ; \\
7. & Else \\
8. & Packet $=$ ACCEPT $\& \&$ send to the Base Station ; \\
9. & \\
10. & Else \\
11. & Packet $=$ NOTACCEPT $!$ \\
12. & \\
13. & \\
\hline
\end{tabular}

Figure 1. Flowchart of the proposed mechanism

Overall, the transmission of error packets without earlier discarding would decrease the chance of non-corrupted packets to be served earlier in the system. Also, this would waste the network resources if the corrupted packets already arrived at the destination but they need to be dropped and retransmitted in the system due to presence of corrupted bits in the packets. This phenomenon would directly influence the network performances in terms of high delay and packets loss in WBAN. Therefore, this study proposed a method which is aimed to curb down the network congestion at the early stage of data transmission. This is done through discarding the corrupted packets before transmission of data is initiated. The performances of this proposed mechanism would be evaluated in terms of delay, packet loss, throughput and packet delivery ratio (PDR). 


\subsection{Experimental Setup}

The simulation of WBAN network is carried out in OMNeT+ simulator [16], [17] using MAC protocol of IEEE 802.15.4 (Zigbee) under CSMA/CA since it offers low data rate and low power usage which is suitable to be adopted in healthcare applications. This simulation is focused on the ECG medical data transmission which follows MIT-BIH database for ECG reading of 360 samples/seconds [18], [19]. Hence, with 30 bytes for every packets under IEEE 802.15 .4 of $250 \mathrm{Kbps}$ would yield packet rate up to 16 packets/second.

The range of packet size from 30 bytes to 60 bytes are considered as suitable and fit for time critical WBAN application [8], [20], [21]. However, value of 30 bytes of data is used in this study because smaller packet size reduces the transmission delay. In contrast, larger packet size increases the delay of data transmission. Also, loss of single packet of large size could bring more severe effect to the performances of the WBAN compares to the small packet size.

This simulation is tested by varying the value of Send Interval of 0.06 seconds (high traffic) and 0.18 seconds (low traffic) under static movement within $50 \mathrm{~m}$ x $50 \mathrm{~m}$ coverage area (typical ward size) as shown in Figure 2. The number of nodes are set with different value range from 20 until 60. The details about other parameters and the used values are simplified in Table 2.

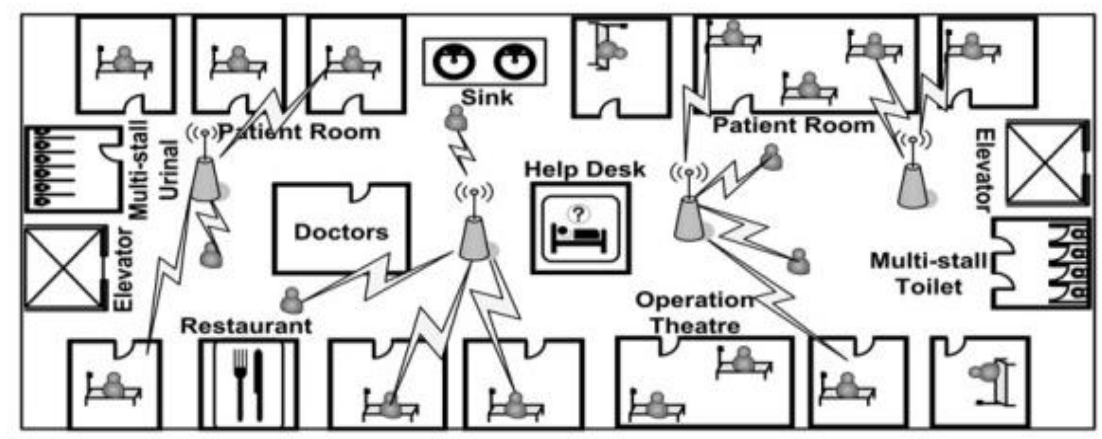

Figure 2. Illustration of WBAN environment [22]

Table 2. Settings of Parameters in WBAN Simulation

\begin{tabular}{lc}
\hline Parameters & Value \\
\hline Packet size & 30 bytes \\
Buffer length & 100 \\
Data rate & $250 \mathrm{Kbps}$ \\
Routing protocol & AODV \\
Simulation time & 200 seconds \\
\hline
\end{tabular}

\section{RESULTS AND ANALYSIS}

In general, this paper is interested in analyzing the effects of earlier discarding of the corrupted packets to the network performances through changing the types of traffics and number of nodes. The measured network performances metrics in this WBAN simulation are listed as follows.

\subsection{Total Delay}

It reflects the sum of delay for all of generated packets in the network from the time of generation until they arrive at the base station. Specifically, the generated packets would face four types of delays such as processing, queueing, propagation and transmission before arriving the base station.

In Figure 3, it is clearly shown that the proposed method yielded less total delay compared to conventional WBAN in both high and low traffics. Generally, the increment of nodes would generate more packets. Therefore, there is a probability that more packets are corrupted. This is especially true when the network is in high traffic which would increase the probability of congestion as well. Transmission of these packets without filtering them first would waste the scarce resources of WBAN such as bandwidth and energy. This is because they still receive same service as non-corrupted packets in the queue. Once they arrived at destination, they will need to be dropped and retransmitted due to corrupted bits in the packets. By discarding the corrupted packets before initiating the transmission would hinder the congestion issue. This leads to lower delay that increases efficiency of data transmission and reception in the WBAN. 


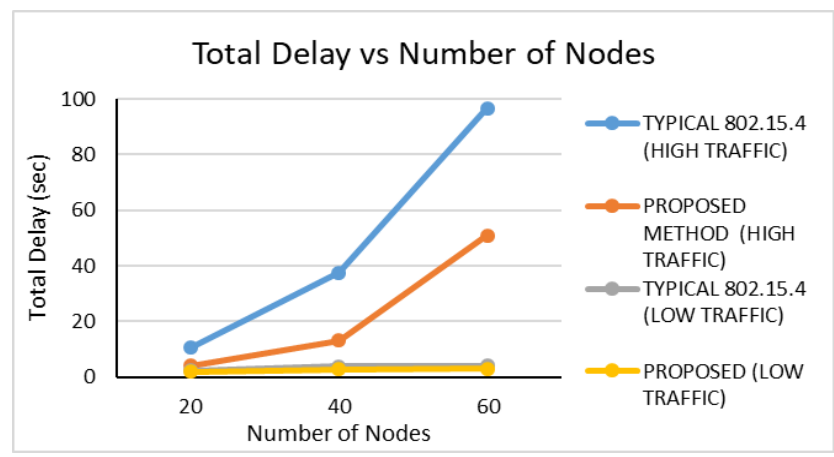

Figure 3. Graph of total delay vs number of nodes

\subsection{Percentage Packet Loss}

It defines the ratio of packets loss to the number of packet sent by the nodes in the network which is shown in 3 as follows

Percentage Packet Loss $(\%)=$

$\frac{\text { Number of sent packets-Number of received packets }}{\text { Number of sent packets }} \times 100$

Referring to Figure 4, the highest rate of packet loss is recorded in typical WBAN for high traffic as number of nodes increases. It is worth to note that there is a significant difference in loss of packets when involving low traffic for proposed mechanism and typical WBAN. This is because in low traffic, less packets are generated and less tendency of congestion occurrence. Also, the chance of packet corrupted is low in the network. Yet, the proposed technique still conquering the performance under higher number of nodes by showing less percentage of the packet loss in the WBAN.

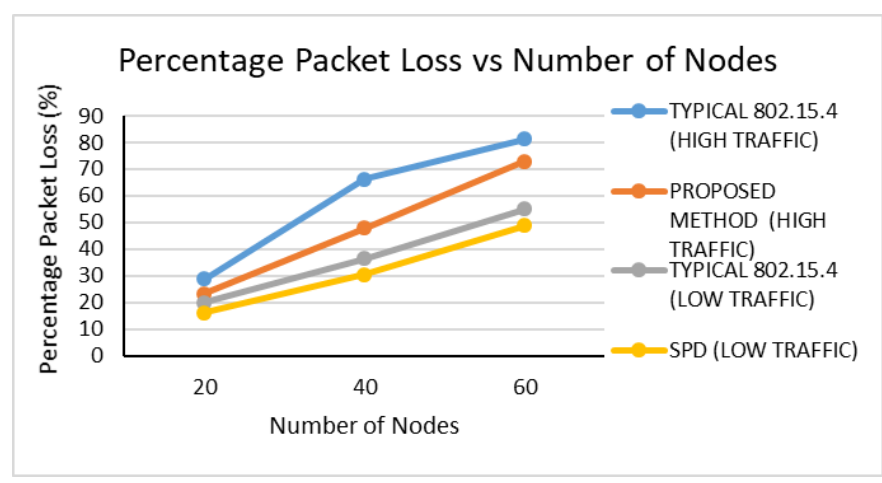

Figure 4. Graph of percentage packet loss vs number of nodes

\subsection{Throughput}

It represents rate of successful bits transferred to the destination over simulation time and it is measured in bits per second (bps) as shown in 4.

$$
\text { Throughput }=\frac{\text { Number of received packets } x 8}{\text { Simulation time }}
$$

Hypothetically, the value of the throughput should be lower in high number of nodes. From Figure 5, high throughput is notified under lower number of nodes for high traffics in the proposed mechanism and ordinary WBAN. The finding from the proposed technique shows better throughput performances compared to conventional WBAN. This shows that high number of successful packets have been received at the destination under high traffic in low number of nodes. 


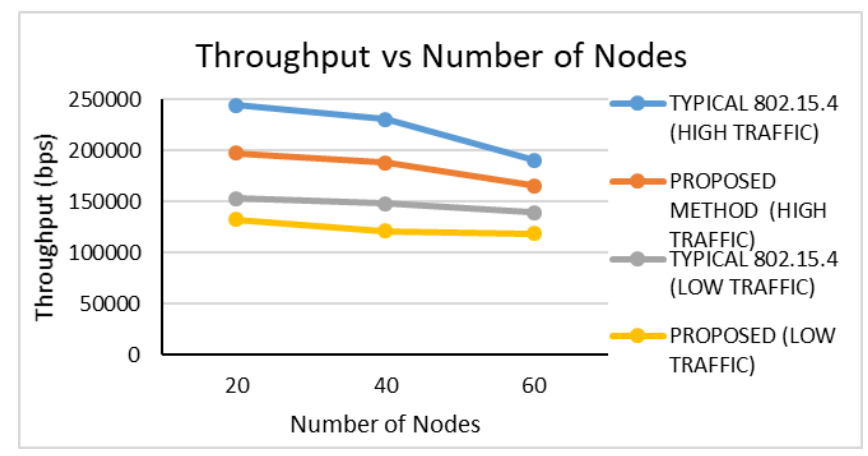

Figure 5. Graph of throughput vs number of nodes

\subsection{Packet Delivery Ratio (PDR)}

It expresses the ratio of number of obtained packets at the base station to the number of sent packets by the nodes as reflected in 5 .

$$
\text { Packet Delivery Ratio }(\mathrm{PDR})=\frac{\text { Number of received packets }}{\text { Number of sent packets }}
$$

As depicted in Figure 6, the PDR is high for low traffic compared to high traffic. This means that high number of packets have successfully received. In low traffics, the congestion phenomenon is less to exist due to low generation of packets. In addition, less probability of packet corrupted in the network. So, more packets are able to be well served in the network. Still, the proposed technique is dominating the performance in high traffic of higher number of nodes by yielding better PDR compared to the normal WBAN.

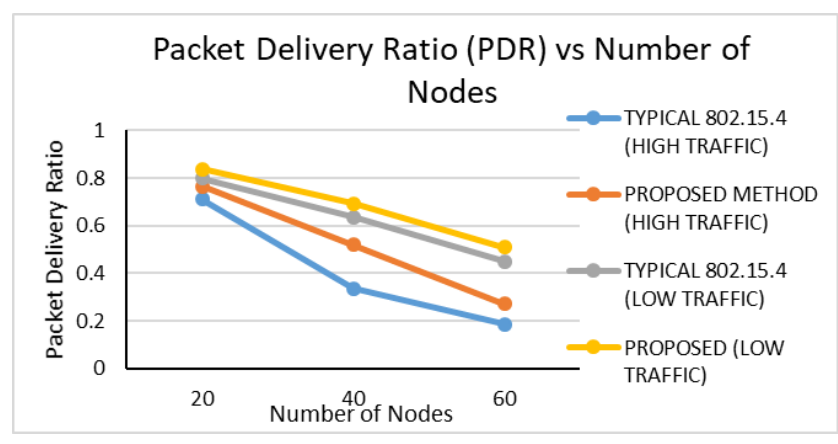

Figure 6. Graph of packet delivery ratio vs number of nodes

\section{CONCLUSION}

The loss of packets especially critical packets due to congestion would severely impact the network performance in the WBAN. In this paper, an earlier discard of corrupted packets has been proposed to attain significant network performances in WBAN of IEEE 802.15.4. The proposed technique outperforms the typical WBAN through decrement of delay, and packet loss, increment in high throughput and PDR under high and low traffics. Although the results show better output, this study will be further enhanced by discarding packets of redundant and obsolete to contribute to greater network performances of WBAN in the future work.

\section{ACKNOWLEDGEMENTS}

The reported research in this paper is financially funded by Ministry of Higher Education (MOHE) Malaysia under Research Acculturation Grant Scheme (RAGS) [Grant number: 9018-00082] and Universiti Malaysia Perlis. 


\section{REFERENCES}

[1] L. Filipe, F. Fdez-riverola, and N. Costa, "Wireless Body Area Networks for Healthcare Applications: Protocol Stack Review," Int. J. Distrib. Sens. Networks, vol. 2015, no. Special Issue on Mobile Sensing and Social Computing, p. 23 pages, 2015.

[2] J. Yusuf Khan, M. R. Yuce, G. Bulger, and B. Harding, "Wireless Body Area Network (WBAN) Design Techniques and Performance Evaluation," J. Med. Syst., vol. 36, no. 3, pp. 1441-1457, 2012.

[3] A. Ghaffari, "Congestion control mechanisms in wireless sensor networks: A survey," J. Netw. Comput. Appl., vol. 52, pp. 101-115, 2015 .

[4] S. Ghanavati, J. Abawaji, and D. Izadi, "A Congestion Control Scheme Based on Fuzzy Logic in Wireless Body Area Networks," in 2015 IEEE 14th International Symposium on Network Computing and Applications, 2015, pp. 235-242.

[5] C. Sergiou, P. Antoniou, and V. Vassiliou, "A Comprehensive Survey of Congestion Control Protocols in Wireless Sensor Networks," IEEE Commun. Surv. Tutorials, vol. 16, no. 4, pp. 1839-1859, 2014.

[6] M. Kathuria and S. Gambhir, "Quality of Service Provisioning Transport Layer Protocol for WBAN system," in 2014 International Conference on Reliability Optimization and Information Technology (ICROIT), 2014, pp. 222-228.

[7] P. Budhwar, B. Sharma, and T. C. Aseri, "Congestion Detection and Avoidance based Transport Layer Protocols for Wireless Sensor Networks," Int. J. Eng. Res. Dev., vol. 10, no. 5, pp. 56-69, 2014.

[8] A. J. D. Rathnayaka and V. M. Potdar, "Wireless Sensor Network transport protocol: A critical review," J. Netw. Comput. Appl., vol. 36, no. 1, pp. 134-146, 2013.

[9] S. Misra, V. Tiwari, and M. S. Obaidat, "LACAS : Learning Automata-Based Congestion Avoidance Scheme for Healthcare Wireless Sensor Networks," IEEE J. Sel. Areas Commun., vol. 27, no. 4, pp. 466-479, 2009.

[10] A. A. Rezaee, M. H. Yaghmaee, and A. M. Rahmani, "Optimized Congestion Management Protocol for Healthcare Wireless Sensor Networks,” Wirel. Pers. Commun., vol. 75, no. 1, pp. 11-34, 2014.

[11] N. Yaakob and I. Khalil, "A Novel Congestion Avoidance Technique for Simultaneous Real-Time Medical Data Transmission," IEEE J. Biomed. Heal. Informatics, vol. 20, no. 2, pp. 669-681, 2016.

[12] Tomáš Ivaniga and P. Ivaniga, "Evaluation of the bit error rate and Q-factor in optical networks," IOSR J. Electron. Commun. Eng., vol. 9, no. 6, pp. 01-04, 2014.

[13] S. M. R. Al Masud, "Study and Analysis of Scientific Scopes, Issues and Challenges towards Developing a Righteous Wireless Body Area Network," Int. J. Soft Comput. Eng., vol. 3, no. 2, pp. 243-251, 2013.

[14] C. Chakraborty, B. Gupta, and S. K. Ghosh, "A Review on Telemedicine-Based WBAN Framework for Patient Monitoring," Telemed. e-HEALTH 619, vol. 19, no. 8, pp. 619-626, 2013.

[15] R. Khalili and K. Salamatian, "A New Analytic Approach to Evaluation of Packet Error Rate in Wireless Networks," in 3rd Annual Communication Networks and Services Research Conference (CNSR'05), 2005, pp. 333-338.

[16] "OMNeT++ Discrete Event Simulator - Home." [Online]. Available: https://www.omnetpp.org/. [Accessed: 23-Feb-2018].

[17] A. Varga and R. Hornig, "AN OVERVIEW OF THE OMNeT++ SIMULATION ENVIRONMENT," in Proceedings of the First International ICST Conference on Simulation Tools and Techniques for Communications Networks and Systems, 2008.

[18] G. B. Moody and R. G. Mark, "The Impact of the MIT-BIH Arrhythmia Database: History, Lessons Learned, and Its Influence on Current and Future Databases," IEEE Eng. Med. Biol., no. June, pp. 45-50, 2001.

[19] K. Kang, "An Adaptive Framework for Real-Time ECG Transmission in Mobile Environments.," Sci. World J., vol. 2014, p. 678309, 2014.

[20] X. Liang and I. Balasingham, "Performance Analysis of the IEEE 802.15. 4 based ECG Monitoring Network," in 7th IASTED International Conferences on Wireless and Optical Communications, 2007, pp. 99-104.

[21] H. S. Yahia and W. M. A. Monnet, "Performance of ZigBee Wireless Body Sensor Networks for Electrocardiogram Signal Transmission under Maximum Payload Size," UKH J. Sci. Eng., vol. 1, no. 1, pp. 19-25, 2017.

[22] S. Misra, J. Mahapatro, M. Mahadevappa, and N. Islam, "Random room mobility model and extra-wireless body area network communication in hospital buildings," IET Networks, vol. 4, no. 1, pp. 54-64, 2015. 